\title{
Critical Condition Detection Using Lion Hunting Optimizer and SVM Classifier in a Healthcare WBAN
}

\author{
Madhumita Kathuria, YMCAUST, Faridabad, India \\ Sapna Gambhir, YMCAUST, Faridabad, India \\ (iD https://orcid.org/0000-0001-5020-8000
}

\begin{abstract}
A timely critical condition detection and early notification are two essential requirements in a healthcare wireless body area network for the correct treatment of patients. However, most of the systems have limited capabilities and so could not detect the exact condition in a precise time interval. In addition to these it needs a reduction in the false alert rate, as issuing alerts for the deviation in each incoming packet increases the false alert rate and these false alerts consume more network resources. In order to fulfill the above-mentioned requirements, a dynamic alert system has been designed in this regard to make it more efficient, also, a new kind of hybridization approach is being introduced to it with the additive support of a nature-inspired optimization strategy named Lion Hunting and a machinelearning technique called support vector machine. The simulation is done using a network simulator NS-2.35, and the proposed alerting system outperforms others.
\end{abstract}

\section{KEYWORDS}

Abnormality Detection, Alert System, False Alerts, Healthcare, Lion Hunting Optimization, Machine Learning, Nature-Inspired Optimization, Support Vector Machine Classifier, Wireless Body Area Network

\section{INTRODUCTION}

Developing of a better alerting system for the correct diagnosis of patient's condition plays a very imperative role in Healthcare Wireless Body Area Network (HWBAN), as false diagnosis leads incorrect decisions, which further causes problems in treatment and even became fatal for patients' life in some cases (Ando et al., 2016; Hauskrecht et al., 2013). In life-critical HWBANs, it is always preferable to transmit accurate data within a specific time interval, as even a slight ignorance in diagnosis can make a significant difference in the decision. The effective fact with the HWBAN is its dynamic nature, where the vital signals change their mode of significance frequently. For an HWBAN system Quality of Service (QoS) means, provisioning of exact diagnosis of the health condition, because any mistake, delay or loss of critical data can become panic for the life of a patient (Kathuria, \& Gambhir, 2014; Salem, et al., 2013; Chaari, \& Kamoun, 2011). However, the study says that most of the alerting systems (Tóth-Laufer, \& Várkonyi-Kóczy, 2014; Skubic et al., 2013) 
are incompetent to identify the exact condition of a patient in the specific instance of time. The main difficulties with these systems are the false alerts notification problem (Salem, Liu, Mehaoua, \& Bautaba, 2014). Received data may be imprecise due to various reasons like a sensor or link error, limited resources, interference during transmission, and transmission error, etc. All of these issues may lead to generate false alerts (Haque, et al., 2015; Dickson, \& Thomas, 2015). Other barriers to these kinds of systems carrying assorted traffic and limited resources (Gambhir, \& Kathuria, 2018) which includes other kinds of obstacles to it. From the above-mentioned analysis it is evident that the HWBAN has a great need of an enhanced model with new thinking so that early and accurate detection of the actual condition can be done within the precise time interval.

Recently, various machine learning methods (Arumugam, \& Jose, 2018, Este, et al., 2009) have fascinated the attention of many researchers in different application areas, and according to them, machine learning approaches can be used for intelligent classification purpose also. On the other hand, according to some other researchers (Dickson, \& Thomas, 2015), nature-inspired algorithms are preferable in many areas due to their robust and bendy nature towards the enhancement of the various unsolved solutions. Concerning these benefits, nowadays different application areas are considering the combined methodology (Eswaramoorthy, et al., 2016) of these two mechanisms for their profit. The understanding as mentioned above encourages us to embed these two mechanisms in our system to make it acquainted with the vital atmosphere of the HWBAN. Therefore, a hybridization model is introduced here which carrying together the benefits of both Lion Hunting (LH) and Support Vector Machine (SVM) techniques and is named as LH-SVM based alerting system (LHSVMAS). Both LH and SVM have tried to make this idea successful by overcoming the remained limitations. The main objective of the LH-SVMAS is to lessen the false alert rate and provide the accurate classification of the critical packets so that they can be transmitted as early as possible with no loss towards the concerned caregiver. Unlike other protocols, it does not require user intervention hence considered as much more effective than others and the simulation outcomes proof the same in a better way. Here, the simulation was conducted in the network simulator NS-2.35.

The rest of the paper is organized as follows. Section 2 provides the related work. Section 3 presents the background details of SVM and LH techniques. Section 4 provides the detail concept of the proposed methodology. Section 5 evaluates the performance of the proposed system. Section 6 offers the conclusion part.

\section{RELATED WORK}

In this section, a brief overview on the work that has been done using LH and SVM described separately. After that, a detailed study of these works which had been taken place till date in regards to an alerting system using a hybridization concept of both nature-inspired and machine learning techniques are explained.

Paper in (Yazdani, \& Jolai, 2016) introduces the use of lion optimization algorithm to accomplish the identification process in the nonlinear systems, especially bilinear system, which further improves the global optimization algorithms for the achievement of good identification precision. A novel optimization algorithm called Lion optimization algorithm was explained by (Wang, et al., 2012; Rajakumar, 2012). Detailed knowledge about lion evolution process and group living theory was brief out in this paper. It describes all the behaviors of lion such as Lifestyle of Pride and Nomad lions, Territorial Marking, Migration, Mating, Territorial Defense and Territorial Takeover, Laggardness Exploitation, etc. It was cleared from the experimental results that lion optimization process outperforms the other algorithms.

An anomaly detection approach for medical WSN was designed by (Salem et al., 2014) which helps to generate a model based on algorithmically located correlations in the data. It uses an SVM classifier which classifies abnormal instances in the incoming sensor data. When required sample found, it applies a periodically rebuilt, regressive prediction model to the abnormal instance to detect 
whether the patient is having a critical condition or the sensor is reporting faulty readings. It has the ability to quickly identify and differentiate between sensor faults and irregularities in a patients' health to preserve robustness and high accuracy in the system. It maintains a higher true positive and lowers false negative rate which further provides high detection accuracy.

An anomaly detection algorithm was designed by (Islam et al., 2018) which has the capability to deal with the common issues of sensor data i.e. incompleteness, ignorance, vagueness, imprecision, and ambiguity etc. It follows an inference mechanism which consists of feature like; input transaction database, conversion into belief transaction database, support calculation, belief matrix, and confidence calculation and belief association rule discovery. It performed better than the other techniques due to the fusion of Gaussian, Binary association rule and Fuzzy association rule, where the Gaussian and Binary association rules can easily segregate true or false values while the fuzzy association rule can handle uncertainty due to vagueness, and ambiguity. This algorithm is capable to predict different expert systems as anomalous data can be removed more efficiently by the above-mentioned rules.

A clinical decision support system for pregnancy care was proposed by (Moreira et al., 2018). Here a particle swarm optimization (PSO) method was applied for reducing the computational cost of the ANN-based method referred to as the multilayer perceptron (MLP), without reducing its precision rate. The PSO algorithm improves computational model performance, showing lower validation error rates than the conventional approaches. It selects the best parameters and provides an efficient solution for training the MLP algorithm. This technique also helps in handling uncertainty in the decision-making process related to high-risk pregnancy. This approach works in a better way in terms of precision and in terms of the true positive ratio (TPR), and showed a reduction in the false positive ratio (FPR). The PSO algorithm helps in optimization of ANN architectures to endow with a low computational cost and excellent precision, but it increases the complexity in the system.

A Multi kernel based dynamic fractional lion optimization algorithm was proposed by (Chander et al., 2017) for data clustering. It was inspired by the lion pride behavior, where the female lion was updated to determine the optimal value based on dynamic directive operative searching strategy. In its first stage, it has used four kernel functions such as Gaussian kernel, Tangential, Rational Quadratic and Inverse multi quadratic kernel function to calculate a fitness function. Then, it has used a fuzzy clustering to evaluate the distance measurement based on above kernel function. In the second phase, a dynamic directive operative searching algorithm was applied into the adaptive fractional lion algorithm which exploits the new fitness function to evaluate the value for the searching agents and to find out the optimal cluster centroid to perform the data clustering. The proposed algorithm achieved higher clustering accuracy which enhanced the clustering performance in the system but increases the computation cost due to the use of four kernel functions.

This study concludes that the SVM classifiers put forward high classification rates in comparison to other classification methods. However, the LH optimization was used for various high-dimensional critical unsolvable problems to offer optimal solutions as compared to rest algorithms. Hence all these factors motivate us to choose a fusion approach of both LH and SVM as core approaches to estimate actual health-status of a patient in an HWBAN.

\section{TECHNIQUES}

\section{Support Vector Machine Classifier}

SVM is a special kind of popular machine learning approach (Zidi et al., 2018) developed for binary classifications. The major characteristics of SVM are the use of the kernel-induced feature spaces, the absence of local minima, and the sparseness of the solution and makes itself suitable for realworld application with a limited amount of training data as it considers only the closest data point as its training set. Most of the classifiers separate classes using a concept of the linear hyperplane. But SVM (Ding et al., 2013; introduced the concept of high-dimension hyperplane separation to a data set that cannot be separated linearly by mapping the train data onto a new, higher dimensional space 
in which they can be separated linearly. Basically, finding the best position for the division plane is an optimization problem which facilitates a kernel function to create linear boundaries through nonlinear transformations. Selection of this separation position of a decision boundary or hyperplane is a key need of SVM classifier to provide the optimum separation point of regions. For any problem, it first breaks the problem into two different classes and for that it selects a margin between the two classes, where the margin is definite to the summation of the distances of the closest data points of the two classes to the hyperplane of SVM. The crucial challenge in SVM (Yuan et al., 2010) is to find a most favorable hyperplane which has the potential to maximize the margin and minimize the number of misclassification errors, whereas the two classes are not linearly separable. Basically, the data points or samples that locate at the two separation boundaries are called the support vectors, and only these training samples are used to measure the margin.

Let $\mathrm{T}=\left\{\left(\mathrm{x}_{1}, \mathrm{y}_{1}\right),\left(\mathrm{x}_{2}, \mathrm{y}_{2}\right), \ldots,\left(\mathrm{x}_{\mathrm{n}}, \mathrm{y}_{\mathrm{n}}\right)\right\}$ is the training set having $\mathrm{n}$ number of observations or sample. Let $\mathrm{x}$ be a feature vector i.e. the input of the SVM, $x \in \mathrm{R}^{\mathrm{N}}$ and $\mathrm{N}$ is the dimension of the feature vector. Let the $y$ be the class i.e. the output of the SVM, y $\in\{+1$ for positive samples, -1 for negative samples \}. The goal of the SVM is to separate the training set into two separable classes, for the same it needs to identify an optimized separating hyperplane, so that it can satisfy the following two requirements; i) maximization of the margin between the two decision boundaries and ii) correct classifications all $\mathrm{x}_{\mathrm{i}}$.

The optimal hyperplane of SVM is symbolized as:

$\left.w^{*} \phi\left(x_{i}\right)+b\right) \geq 0$

and the classification discriminate is represented by Equation (1):

$y_{i}\left(w^{*} \phi\left(x_{i}\right)+b\right) \geq 1$

where $\mathrm{w}$ denotes the optimal weight, $\mathrm{b}$ denotes the optimal bias, and $\phi$ is the nonlinear mapping function applied to input vectors.

An optimal hyperplane can be obtained from the maximum margin can be calculated from the Equation (2), the optimum weight vector w and bias b:

$$
M=\operatorname{minimize} \frac{\|w\|^{2}}{2}
$$

where $\|w\| l$ is the norm of $w$.

The Equation (1) is representing a convex quadratic programming problem with linear inequality constraints, which is very hard to solve due to inequality constraints problem. So, the concept of Lagrange multipliers is applied to replace these inequality constraints and the equation is restructured into the following equation:

maximize $: L(w, b, \alpha)=\sum_{i=1}^{N} \alpha_{i}-\frac{1}{2} \sum_{i, j=1}^{N} \alpha_{i} \alpha_{j} y_{i} y_{j}\left(\phi\left(x_{i}\right) * \phi\left(x_{j}\right)\right)$

This provides a quadratic optimization problem. 
It is not always feasible that the training data samples are not linearly separable in a feature space, but with an appropriate transformation of these data sample into a high-dimensional space, an SVM can classify the data into two separable classes. This can be done with a tool known as the Kernel function. With an appropriate choice of the kernel function, the linear non-separable training set can be transferred into one that is linearly separable in the feature set. So, the Equation (3) is transformed to Equation (4):

maximize $: L(w, b, \alpha)=\sum_{i=1}^{N} \alpha_{i}-\frac{1}{2} \sum_{i, j=1}^{N} \alpha_{i} \alpha_{j} y_{i} y_{j} K\left(x_{i}, x_{j}\right)$

subject to:

$$
\begin{aligned}
& \sum_{i=1}^{N} \alpha_{i} y_{i}=0 \\
& \text { Const } \geq \alpha_{i} \geq 0, i=1,2, \ldots . . N
\end{aligned}
$$

where $\alpha$ is the Lagrangian multiplier, Const is the Upper bound constant and $\mathrm{K}\left(\mathrm{x}_{\mathrm{i}}, \mathrm{x}_{\mathrm{j}}\right)$ is the RBF kernel function.

Assuming that $\alpha_{\mathrm{i}}$ is the solution of the problem, then the optimal solution for $\mathrm{w}$ and $\mathrm{b}$ is calculated as follows:

$$
\begin{aligned}
& w=\sum_{i=1}^{N} \alpha_{i} y_{i} x_{i} \\
& b=\frac{1}{N} \sum_{i=1}^{N}\left(y_{i}-\sum_{j=1}^{N} \alpha_{j} y_{j} K\left(x_{i}, y_{i}\right)\right)
\end{aligned}
$$

The SVM classifier is having the potential to resolve the multi-class classifications, with the facility of linear separations by considering its optimal hyperplane. A tool called kernel trick used by SVM is capable to classify non-linear and non-separable samples by transforming the linear space into high-dimensional space.

\section{Lion Hunting Optimizer}

Lion Optimization algorithm (Yazdani \& Jolai, 2016) basically based on simulation of the solitary and cooperative behaviors of lions such as Territorial marking, Roaming, Convergence, Migration, Fighting, Mating, Defense and Takeover etc. These characteristics are projected to find and replace the worst solution to the new best solution. In addition to these, it illustrates a unique behavior entitled lion hunting where the hunters follow a very different style during encircling, attacking and capturing of prey. According to experimental results, the lion optimization technique provides better results for the achievement of fast convergence and global optima for various popular functions as compared to other nature-inspired algorithms as it follows a special kind of learning mechanism called oppositionbased learning (OBL) mechanism (Tizhoosh, 2005) which decreases the local solution and helps to find the global solution within less time. 
Lion hunting optimizer mimics the communal guidance and hunting behavior of lions in the natural world. For hunting, the female members of particular pride or residential group are only taking part in hunting and it's their responsibility to provide food to the whole group members. So, in the first phase of LH optimization algorithm, a selected amount of lionesses are elected for the hunting. During the practice phase of hunting, individual lionesses are selected one after another randomly and put into three different categories (i.e., Left group, Middle group and Right group). The LH algorithm is encompassed with the following control parameters such as groups, the position of prey, the position of hunter lioness, the difference between the position of prey and the position of the hunter, and the chance of increment rate.

Lion hunting optimization algorithm basically follows following steps for optimization:

Step 1: In the Initialization of population phase, the hunting group size which gives the information about a number of solution vectors in the search space is selected. On the basis of the number of hunters and their roles, hunters are randomly assigned to three subgroups: middle-subgroup, left-subgroup, and right-subgroup.

Step 2: The initial position of the victim is estimated using Equation (7). Each selected hunter attacks the target according to their role and group they belong to:

$V_{0}=\left(\sum_{n=1}^{N} H L_{n}\right) / N$

where $\mathrm{V}_{0}$ is the initial position of the victim, $\mathrm{HL}_{\mathrm{n}}$ is initial position of the $\mathrm{n}^{\text {th }}$ hunter lioness, $\mathrm{N}$ denotes a total number of lioness participated in hunting.

Step 3: During hunting, the actual position of each hunter lioness is changed according to the requirement; hence the victim also updates its position according to the situations. If the current position of hunter lioness is better than the previous position, then there is a chance of capturing the victim, otherwise, the victim will run away and the new position of the victim will be obtained by using Equation (8):

$$
V_{i}^{k+1}=V_{i}^{k}+C^{*} r(0,1) *\left(V_{i}^{k}-H L_{i}^{k}\right)
$$

where $\mathrm{V}^{\mathrm{k}+1}$ denotes the new position of the victim, $\mathrm{V}^{\mathrm{k}}$ is the current position of victim, $\mathrm{HL}^{\mathrm{k}}$ is the current position of hunter lioness at $\mathrm{k}^{\text {th }}$ iteration, from $\mathrm{i}^{\text {th }}$ direction as lioness attacks from multiple or $\mathrm{N}$-dimensional directions (both front and opposite directions), $\mathrm{r}$ (Low, High) is defined as the random number generation function which generates a random number in between low and high range. $\mathrm{C}$ defines the chance of increment in the fitness function.

Step 4: At the time of encircling the victim by each individual group, the position of each lioness is updated. The new positions of lioness belong to the middle sub-group is calculated using Equation (9):

$$
H L_{i}^{k+1}=\left\{\begin{array}{l}
r\left(H L_{i}^{k}, V_{i}^{k}\right), \text { if } H L_{i}^{k}<V_{i}^{k} \\
r\left(V_{i}^{k}, H L_{i}^{k}\right), \text { if } H L_{i}^{k}>V_{i}^{k}
\end{array}\right.
$$


where $\mathrm{V}^{\mathrm{k}}$ is the current position of the victim, $\mathrm{HL}^{\mathrm{k}}$ is current position Lioness and $\mathrm{HL}^{\mathrm{k}+1}$ is the new position of Lioness.

Step 5: The new positions of lionesses which are belongs both left and right sub-groups are calculated as follows:

$$
H L_{i}^{k+1}=\left\{\begin{array}{l}
r\left(\left(2^{*} V_{i}^{k}-H L_{i}^{k}\right), V_{i}^{k}\right), \text { if }\left(2^{*} V_{i}^{k}-H L_{i}^{k}\right)<V_{i}^{k} \\
r\left(V_{i}^{k},\left(2^{*} V_{i}^{k}-H L_{i}^{k}\right)\right), \text { if }\left(2^{*} V_{i}^{k}-H L_{i}^{k}\right)>V_{i}^{k}
\end{array}\right.
$$

where $2^{*} \mathrm{~V}^{\mathrm{k}}$ is the current position of the victim from opposite direction, $\mathrm{HL}^{\mathrm{k}}$ is the current position Lioness and $\mathrm{HL}^{\mathrm{k}+1}$ is the new position of Lioness.

Step 6: With time lionesses are revising their position to get another chance to catch the victim. It means the changes in positions of all of the hunters are continuing until the obtained result of the objective function is not satisfied and the exit condition is not convinced.

Step 7: All the above steps are repeated until stopping criteria is achieved.

The key benefit of LH optimizer is that it is capable to find a better global solution within less amount of time, and this is possible because of the OBL technique used by it during hunting which provides fast convergence and reduces local optimal solutions.

\section{PROPOSED LH-SVM BASED ALERTING SYSTEM}

An HWBAN is consists of a patient carrying tiny devices for capturing vital signs from the various body parts of a patient called sensor node. Sensed data from the entire sensor nodes are collected by their head called Central Node $(\mathrm{CN})$. It performs all kinds of pre-processing tasks before transmitting the processed data towards the medical server, but the first and foremost job of an HWBAN is to analyze and notify the actual condition of a patient as early as possible.

Authors in (Haque et al., 2015) had tried to fulfill these requirements by designing a Sensor Anomaly Detection system (called SAD) for a healthcare wireless sensor network. This system predicts the actual condition of a patient by considering past data. It basically compares the actual sensed value against the past stored data and identifies the error. It further used a concept of dynamic threshold value followed by a majority voting to segregate positive alarm from the negative alarm or to identify the inaccuracy. Here the measured value of a physiological parameter is compared with the predicted value for corresponding sensor node to estimate the error and this error is compared against the dynamic threshold value to set the status of 1 or 0 indicating that the parameter is anomalous or normal. These status values are forwarded for voting and according to the majority of voting a decision is made whether the sensor value is correct or not. It reduces the false alert rate and generates true alerts, but it consumes more resources and time when frequently changes occur in the health status of a patient. It did not provide a clear concept of how it estimates dynamic threshold from standard deviation and which factor are considered for the same. The concept of majority voting increases complexity in terms of computation and time when the number of sensor nodes increases in the system.

In order to overcome the abovementioned issues, a Dynamic Alerting System (DAS) was proposed in (Gambhir \& Kathuria, 2018) for the HWBAN. This protocol was designed for the detection and notification of the emergency situation as early as possible. Its eminent detection methodology lessens the false rates and helps in making it very useful for early identification. This keeps a tally of received measurement, calculates the deviation between sense value and the predefined vital sign range of a sensor node. This deviation quantifies the actual condition of a sensor node and also helps to take 
specific actions. Rather than considering each measurement, it considers a group of measurements and for that, it follows a concept of the counter. When it detects a true deviation in the sensed value it set a counter and until this counter become 0 , it waits and monitors that number of incoming packets and find their deviation values. Then it uses these deviation values to estimate the statistical standard deviation value and compared statistical standard deviation against the threshold value (defined by the concerned caregiver) to make a decision i.e. whether the sensor is in normal or abnormal condition. It works better than the SAD protocol and successfully reduces the false alert rate, but needs further upgrading to get healthcare application-specific QoS requirements. The concept of counter further adds a delay in the system. When the number of nodes increases in the system the packets loss rate also gets increased and with this loss the waiting time of the system gets increased as it waits for further calculation of standard deviation and which was not possible until the counter becomes 0 and the counter will not come to 0 until the system monitor that number of packets. These kinds of situations increase delay in the processing and degrade system performance.

A new kind of alerting system is designed here for the timely diagnosis of the actual condition of a patient. Contrast to counter in DAS, it uses a timer concept to monitor and measure multiple packets for the estimation of true alerts. Unlike others, it will not send the alerts for each deviation in the sensed value. Instead, it waits, monitors and analyses the deviation in all received packets for a particular time period, calculates the weighted standard deviation and then compares this weighted standard deviation with respect to the critical threshold value for the final decision. It further helps the caregiver to differentiate between very critical and likely to be critical conditions. To do the separation between true alerts and false alerts it uses a hybrid technique called LH-SVM, where the SVM acts as the classifier who classifies true alerts and false alerts into two separate classes and the LH acts as the optimizer which optimizes the kernel parameter and regularization parameter to generate an optimal hyperplane with maximum margin for the SVM.

The working principle of the LSSVMAS is explained as follows.

A critical threshold based alert mechanism is provided here to make it suitable for false alert detection. For accurate condition detection, data of the arrived packets are examined using a statistical method called Weighted Standard Deviation (WSD), where weight defines the frequency of occurrence. Both the WSD and the critical threshold act as two key parameters for the segregation of false alerts from true alerts.

Phase 1: For a particular time interval an analysis of incoming packets was conducted in the first phase where it keeps a count of received packets, and checks and estimates the actual deviation (AD) in the measurement of the vital signal (i.e. calculated by subtracting sensed value from the actual range of a vital signal). This $\mathrm{AD}$ value is further used to recognize the real criticality level of a patient. The formula for the calculation of $\mathrm{AD}$ is given in Equation (11):

$$
A D= \begin{cases}\text { Measure }_{\text {value }}-\text { Upper }_{\text {range }}, & \text { if Measure } \\ \text { Lower }_{\text {range }}-\text { Measure }_{\text {value }}, & \text { if Measure } \text { Ualue }_{\text {vange }}<\text { Lower }_{\text {range }} \\ 0, & \text { if Lower } \text { range }_{\text {rasure }} \leq \text { Mealue }_{\text {valuer }} \leq \text { Upper }_{\text {range }}\end{cases}
$$

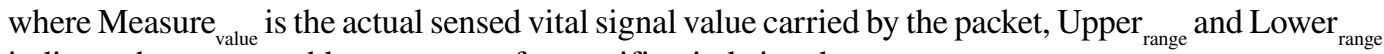
indicate the upper and lower range of a specific vital signal.

Phase 2: It provides an additional concept called activation of the critical field to make aware a caregiver about the low-level criticality, not the higher one. Because of this concept, the proposed system can successfully detect and report the actual situation in all circumstances. The job of critical field is to inform the medical server about the likely to be critical condition so that when 
the medical server receives a packet with critical field in active mode, then it will not send an alert message to the caregiver, but at the same time, this field helps the medical person to diagnose the pre-critical condition of a patient. It repeatedly monitors each incoming packet and keeps a count of the number of the received packet using a variable named Counter unless until the time interval becomes zero, then estimates the $\mathrm{AD}$ values and stores $\mathrm{AD}$ values into $\mathrm{CN}$ 's database. According to the calculated value of the $\mathrm{AD}$, it activates the critical field of the received packet by following the Equation (12):

$$
\text { Critical_field }= \begin{cases}1, & \text { if } A D !=0 \\ 0, & \text { otherwise }\end{cases}
$$

where $\mathrm{AD}$ is the actual deviation found in the packet.

Phase 3: When the time interval expired, it reads all ADs from CN's database and arranges them in an ascending order. After that, it finds the WSD value using the concept of a statistical median calculus as given in Equation (13):

$$
W S D=\sqrt{\frac{\sum_{i=1}^{\text {Counter }} f r_{i} * v^{2}}{\sum_{i=1}^{\text {Counter }} f r_{i}}-\left(\frac{\sum_{i=1}^{\text {Counter }} f r_{i} * v}{\sum_{i=1}^{\text {Counter }} f r_{i}}\right)^{2}}
$$

where WSD denotes the weighted standard deviation, fr denotes the frequency or number of repetitions of the $\mathrm{AD}, \mathrm{v}$ denotes the difference in the $\mathrm{AD}$ and the median value, and Counter denotes the number of received packets at that time interval.

Phase 4: An additional field named Alert field of a packet plays a very important role to assists the medical server to take the correct decision in regard to true and false alerts.

Here the SVM classifier comes into the scenario. The task of this classifier to separate true alerts from false alert i.e. a model $(x ; y)$, where $x=[W S D$, Critical_Threshold, Counter, Timer $]$ as input training sets and $\mathrm{y}=[$ Alert_field $=1$, Alert_field $=0]$ as true and false alert. There are various kinds of kernel function are present for SVM, but here a Radial Bias Function (RBF) is considered as the kernel function as given in Equation (14) for the transformation the non-separable data to a higher dimensional space, essentially yielding high-level flexibility in the decision boundary, resulting in substantial performance enhancement:

$$
K\left(x_{i}, x_{j}\right)=\exp \left(-\frac{\left|x_{i}-x_{j}\right|^{2}}{2^{2}}\right)
$$

Now the LH optimization algorithm is applied to optimize the kernel parameters as well as regularization parameter so that an optimal hyperplane with a maximum margin can be decided for the SVM classifier.

After the selection of optimal hyperplane, the SVM classifier extracts all the useful data samples called support vectors from the training sets who can take part in the testing sets and these support 
vectors help to identify whether the Alert_field will feed with ' 1 ' or ' 0 ' values. The parameters WSD and Critical_Threshold act as the base for this classification. The formula is given in Equation (15) helps in identifying accurate decision by the classifier:

$$
\text { Alert_field }= \begin{cases}1, & \text { ifWSD }>\text { Critical_ Threshold } \\ 0, & \text { otherwise }\end{cases}
$$

where WSD denotes the weighted standard deviation and Critical_Threshold denotes the critical threshold value.

Now the CN checks the Alert_field, if its value is 1 , then it assigns a highest priority to it and serves it on priority basis earlier than other packets. Finally, it updates its database.

Phase 5: When the medical server received a packet with an alert field in active mode, it immediately issues an alert message and sends it to the concerned caregiver, so that he/she can take further useful decisions.

The workflow diagram for whole LH-SVM based alerting system is explained in Figure 1. Algorithm 1 presents the pseudo-code for the proposed alerting system.

Figure 1. The workflow diagram whole LH-SVM based alerting system

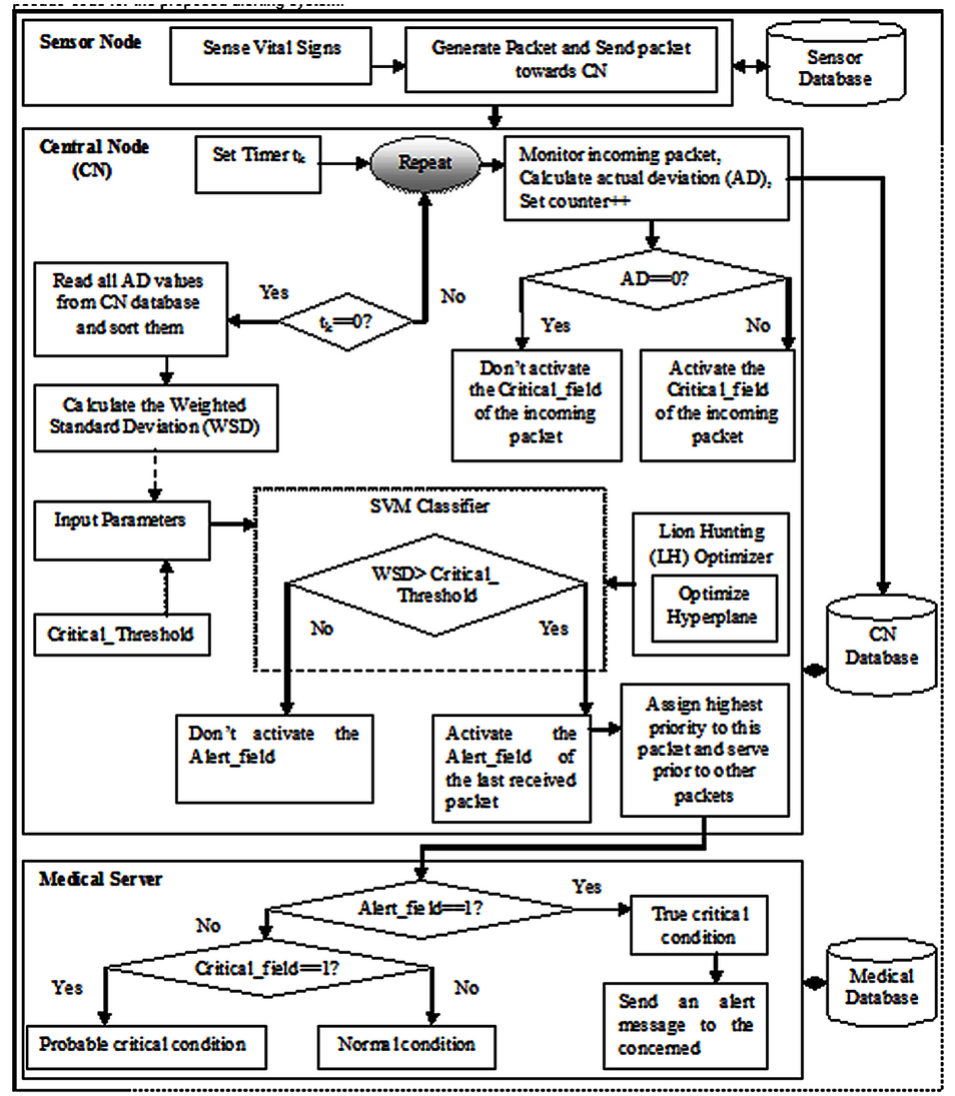


Algorithm 1. Pseudo-code for LH-SVM based alerting system

PROCEDURE: Abnormality detection and notification

Input: Packet

Output: True alerts/False alerts

1. Initialize Time interval $\left(t_{k}\right)$, received counter i.e. Counter=0

2. Repeat

3. Received a packet

4. Set Counter++

5. Monitor sensed value

6. Find actual deviation(AD)

7. Store value of $\mathrm{AD}$ in $\mathrm{CN}^{\prime} \mathrm{s}$ database

8. If $A D !=0$ Then

9. Activate the critical field this packet of $t$ i.e. critical field=1 10. Else

11. Do not activate the critical_field this packet of $t$ i.e. critical_field=1

12. Until timer expired

13. Read all AD values from the database

14. Sort them into ascending order

15. Estimate the weighted standard deviation (WSD) using Equation (13)

16. Read the Critical Threshold value

17. Alert_field= CALL SVM_classifier(WSD, Critical_Threshold, Counter, $t_{k}$ )

18. If Alert field! $=0$ Then

19. Notification of false alert

20. Else

21. Notification of true alert

22. Assigns highest priority to the current received packet

23. Send this packet prior to other packet towards medical server

24. Update $\mathrm{CN}^{\prime} \mathrm{s}$ database

PROCEDURE: SVM_classifier (WSD, Critical_Threshold, Counter, $t_{k}$ )

Input: Training data

Output: Activate/ Deactivate Alert_field

1. For time interval $t_{k}$

2. hyperplane=CALL LH_optimizer (range of RBF kernel parameters, range of regularization parameter)

3. Choose the relevant training set

4. Run the RBF kernel function on these training set using the optimal hyperplane

5. Set the current packet's Alert_field i.e. $\{1,0\}$

6. Return the value of Alert fielo

PROCEDURE: LH_optimizer (range of RBF kernel parameters, range of regularization parameter)

Input: Range of RBF kernel parameters, range of regularization parameter Output: Optimal hyperplane

1. For time interval $t_{k}$

2. Randomly generate a population of hunter lioness

3. According to their hunting ability segregate these lions into three sub-groups i.e. middle, left and right

4. Estimate the initial position of the victim or the prey using Equation (7) 


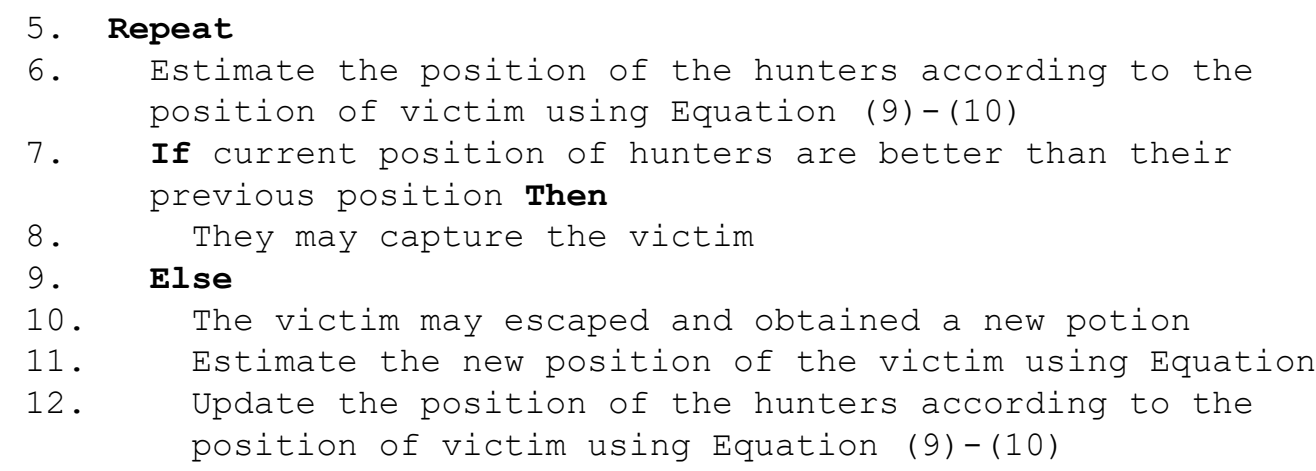

13. Until the termination criteria reach.

The proposed LHSVMAS approach tries its best to optimize the SVM classifier accuracy by estimating the best values of the regularization and RBF kernel parameters. In order to achieve the same, this approach is applied with the LH optimizer. The use of LH maximizes the margin (i.e., separation width) which further helps in reducing the number of weights that are nonzero. This also acts as an important factor during the decision-making of the separating line (hyperplane) as these nonzero weights directly corresponds to the support vectors, because they straightly support the separating hyperplane. The LH method obtained the optimal margin very quickly only because of its features i.e. fast and rapid convergence towards global solutions. The use of SVM with RBF kernel reduces the misclassification rate and classification time complexity. The application of both LH and SVM techniques in LHSVMAS make it a very powerful tool for classification of true and false alerts and enhance the quality of abnormality detection and early notification.

\section{PERFORMANCE EVALUATION}

The proposed model is evaluated by means of above study to differentiate the performance gap between SAD, DAS and itself. The effectiveness of the proposed LHSVMAS is actually improved because of combined application of both LH optimization and SVM classification. Here in order to judge the performance capability of each one various QoS metrics i.e. loss, delay, and throughput are compared and evaluated. The generated results show the efficiency of both LH and SVM in this regards. The implementation of all these three alerting systems are conducted in the ns- 2.35 network simulator which evaluates their potential with respect to various QoS metrics and a MATLAB tool is used to generate the performance evaluations graphs for each QoS metrics. The simulation is carried out for an increase in the number of sensor node so that the efficiency of each system can be measured in high as well as low load.

\section{Packet Loss Ratio}

The Packet Loss Ratio (PLR) is calculated as the ratio of a total number of packet losses with respects to a total number of packets generated from various sources. The PLR is calculated at the central node using Equation (16). The performance evaluation of PLR metric is outlined in Figure 2:

$$
\text { PLR }=\frac{\text { Total number of packets lost }}{\text { Total number of packets sent }} * 100
$$

Figure 2 illustrates that LHSVMAS outperforms both SAD and DAS for an increase in load; however DAS also performs in a more proficient way as compared to SAD and has a $36 \%$ reduction 
Figure 2. Performance evaluation graph for packet loss ratio

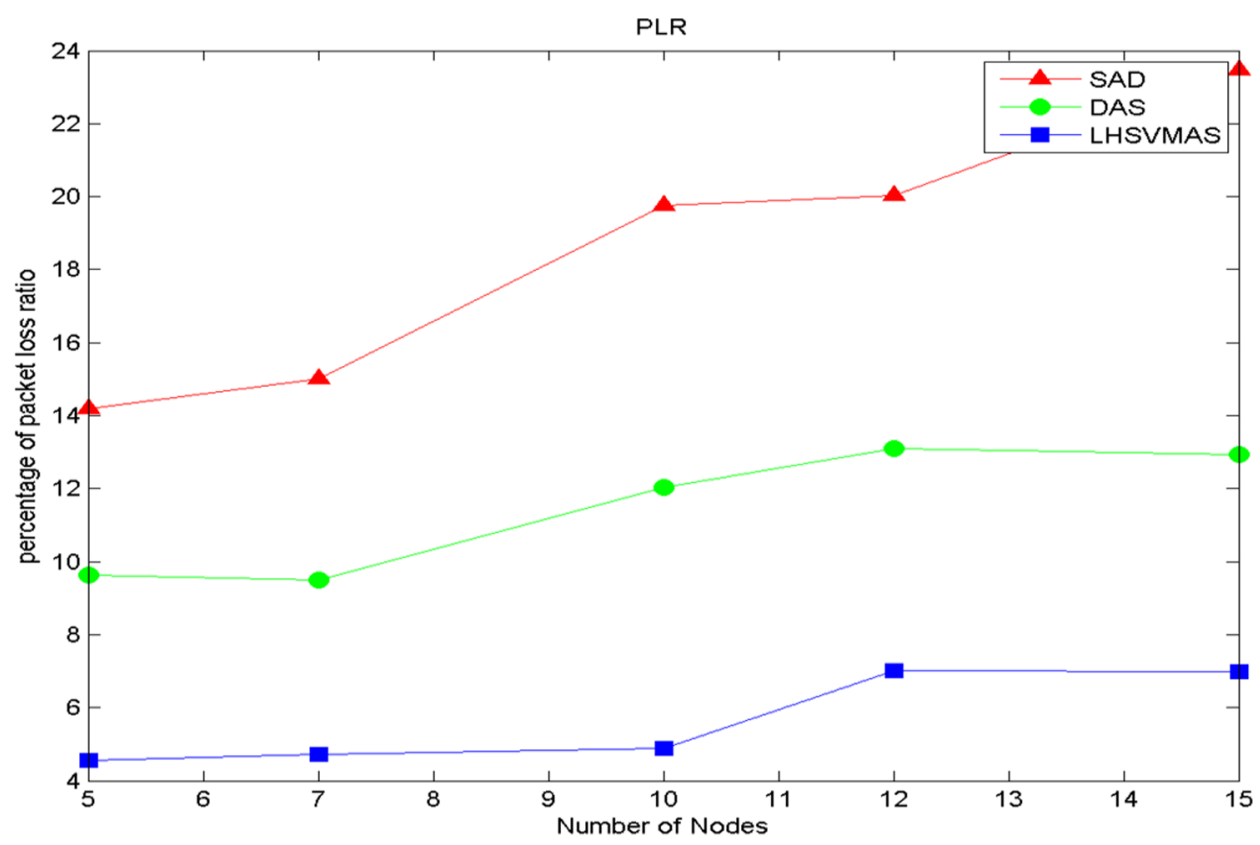

in the loss with respect to SAD. As said earlier LHSVMAS is used to minimize the loss rate and to improve throughput, it has a nearly $51 \%$ reduction in loss in contrast to DAS.

\section{Transmission Delay}

The transmission delay is defined as the total time taken by the packet to travel from a sender sensor node to the receiver or $\mathrm{CN}$. That means the total amount of time consumed for successful delivery of a packet at the $\mathrm{CN}$. The formula for the calculation of transmission delay is given in Equation (17). Figure 3 presents the performance evaluation graph for the transmission delay metric:

Transmission Delay $=\frac{\text { Total elapsed time during packet transmission }}{\text { Total number of packets sent }- \text { Total number of packets lost }} * 100$

From Figure 3 it is clear that LHSVMAS provides better results in terms of transmission delay than other two. The delay for LHSVMAS goes down as the number of nodes increase. DAS acquires less delay i.e. a percentage of $14 \%$ less than the SAD, while the LHSVMAS provides delay with a reduction rate of $31 \%$ against DAS.

\section{Throughput}

Throughput defines the total number of packets delivered successfully at the $\mathrm{CN}$ during the whole simulation. The formula for throughput calculation is given in Equation (18). Figure 4 shows the Performance evaluation graph for the throughput:

Throughput $=\frac{(\text { Total number of packets delivered }) *(\text { packet size }) * 8}{\text { Total Simulation Time }}$ 
Figure 3. Performance evaluation graph for transmission delay

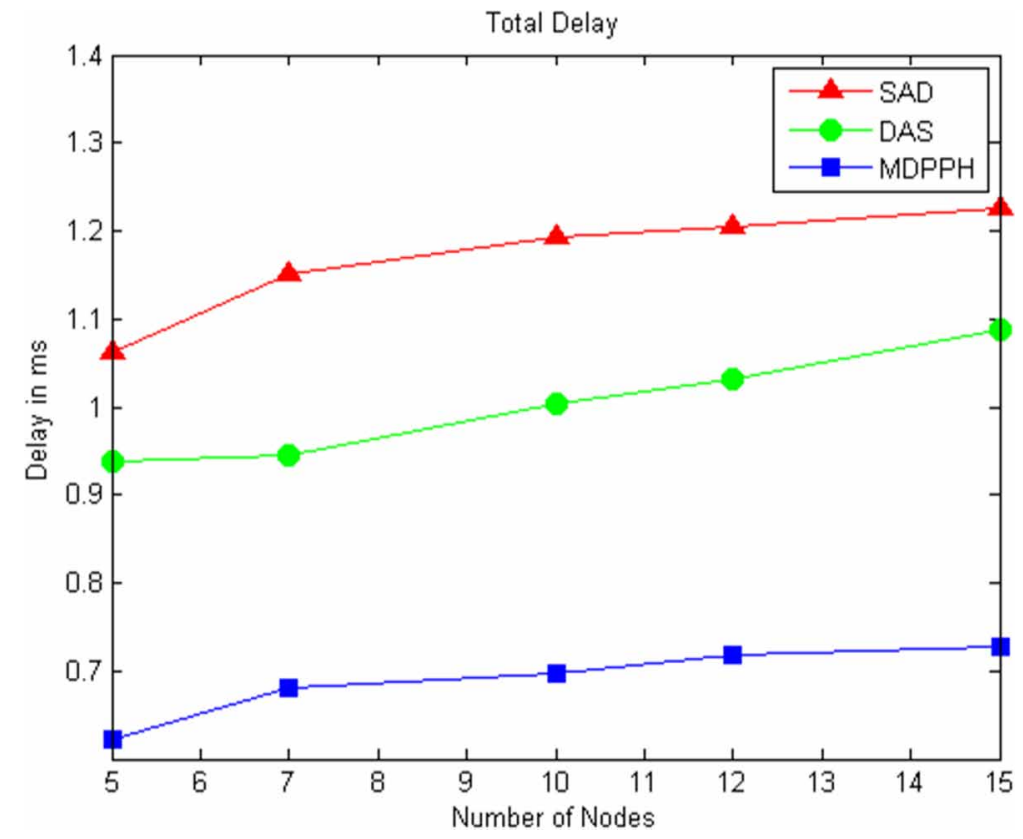

Figure 4 provides the total throughput results. The performance of throughput degrades when loss and delay rate increases. LHSVMAS scored higher throughput against DAS and SAD as it reduces loss and delay rate. The LHSVMAS has nearly $40 \%$ amplification in throughput as compared to DSA and the DAS has $25 \%$ improvement in throughput as compared to the SAD.

Figure 4. Performance evaluation from network Throughput

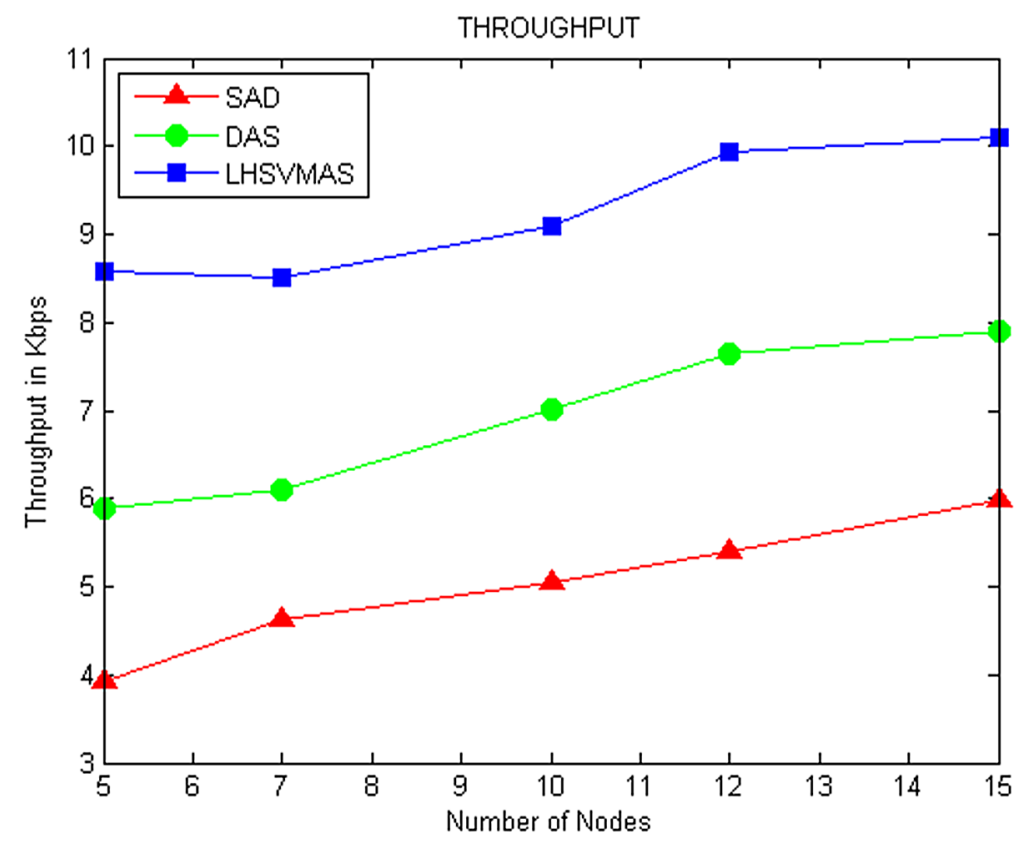




\section{CONCLUSION}

In this paper, a hybrid technique for critical condition classification and detection is presented and compared. The pillar of this technique totally depends on a nature-inspired and machine learning mechanism. In its first stage, it uses a lion hunting technique for optimization of hyperplane for SVM. In its next stage, it uses the SVM classifier to identify true alerts. It is found that LH optimization works very effectively for finding the optimal margin due to its prompt convergence towards global solutions. Put one step ahead, the SVM put its efforts for successful classification of true alert and false alerts which further helps in removing the false alert transmission from the system. The proposed alerting system is evaluated and compared against both DAS and SAD. All these three approaches (i.e., SAD, DAS, and LHSVMAS) were experimentally evaluated by very well-known QoS performance metrics i.e., Loss, Delay, and Throughput. Results from the simulation show the efficiency of LHSVMAS, as it outperforms others due to its hybrid optimization and classification mechanisms. 


\section{REFERENCES}

Andò, B., Baglio, S., Lombardo, C. O., \& Marletta, V. (2016). A multisensor data-fusion approach for ADL and fall classification. IEEE Transactions on Instrumentation and Measurement, 65(9), 1960-1967. doi:10.1109/ TIM.2016.2552678

Arumugam, P., \& Jose, P. (2018). Efficient Decision Tree Based Data Selection and Support Vector Machine Classification. Materials Today: Proceedings, 5(1), 1679-1685.

Chaari, L., \& Kamoun, L. (2011). QoS concepts and architecture over wireless body area networks for healthcare applications. International Journal of E-Health and Medical Communications, 2(4), 50-66. doi:10.4018/ jehmc.2011100104

Chander, S., Vijaya, P., \& Dhyani, P. (2017). Multi kernel and dynamic fractional lion optimization algorithm for data clustering. Alexandria Engineering Journal.

Dickson, A., \& Thomas, C. (2015, February). Optimizing false alerts using multi-objective particle swarm optimization method. In Proceedings of the 2015 IEEE International Conference on Signal Processing, Informatics, Communication and Energy Systems (SPICES) (pp. 1-5). IEEE. doi:10.1109/SPICES.2015.7091547

Ding, L., Yu, F., Peng, S., \& Xu, C. (2013). A Classification Algorithm for Network Traffic based on Improved Support Vector Machine. Journal of Computers, 8(4), 1090-1096. doi:10.4304/jcp.8.4.1090-1096

Este, A., Gringoli, F., \& Salgarelli, L. (2009). Support vector machines for TCP traffic classification. Computer Networks, 53(14), 2476-2490. doi:10.1016/j.comnet.2009.05.003

Eswaramoorthy, S., Sivakumaran, N., \& Sekaran, S. (2016). Grey wolf optimization based parameter selection for support vector machines. COMPEL, 35(5), 1513-1523.

Gambhir, S., \& Kathuria, M. (2018). Dynamic priority-based packet handling protocol for healthcare wireless body area network system. International Journal of Computational Systems Engineering, 4(1), 3-16. doi:10.1504/ IJCSYSE.2018.090635

Haque, S. A., Rahman, M., \& Aziz, S. M. (2015). Sensor anomaly detection in wireless sensor networks for healthcare. Sensors (Basel), 15(4), 8764-8786. doi:10.3390/s150408764 PMID:25884786

Hauskrecht, M., Batal, I., Valko, M., Visweswaran, S., Cooper, G. F., \& Clermont, G. (2013). Outlier detection for patient monitoring and alerting. Journal of Biomedical Informatics, 46(1), 47-55. doi:10.1016/j.jbi.2012.08.004 PMID:22944172

Islam, R. U., Hossain, M. S., \& Andersson, K. (2018). A novel anomaly detection algorithm for sensor data under uncertainty. Soft Computing, 22(5), 1623-1639. doi:10.1007/s00500-016-2425-2

Kathuria, M., \& Gambhir, S. (2014, February). Quality of service provisioning transport layer protocol for WBAN system. In Proceedings of the 2014 International Conference on Optimization, Reliability, and Information Technology (ICROIT) (pp. 222-228). IEEE. doi:10.1109/ICROIT.2014.6798318

Moreira, M. W., Rodrigues, J. J., Kumar, N., Al-Muhtadi, J., \& Korotaev, V. (2018). Nature-Inspired Algorithm for Training Multilayer Perceptron Networks in e-health Environments for High-Risk Pregnancy Care. Journal of Medical Systems, 42(3), 51. doi:10.1007/s10916-017-0887-0 PMID:29392487

Rajakumar, B. R. (2012). The Lion's Algorithm: A new nature-inspired search algorithm. Procedia Technology, 6, 126-135. doi:10.1016/j.protcy.2012.10.016

Salem, O., Guerassimov, A., Mehaoua, A., Marcus, A., \& Furht, B. (2013, June). Sensor fault and patient anomaly detection and classification in medical wireless sensor networks. In Proceedings of the 2013 IEEE International Conference on Communications (ICC) (pp. 4373-4378). IEEE. doi:10.1109/ICC.2013.6655254

Salem, O., Guerassimov, A., Mehaoua, A., Marcus, A., \& Furht, B. (2014). Anomaly detection in medical wireless sensor networks using SVM and linear regression models. International Journal of E-Health and Medical Communications, 5(1), 20-45. doi:10.4018/ijehmc.2014010102

Salem, O., Liu, Y., Mehaoua, A., \& Boutaba, R. (2014). Online anomaly detection in wireless body area networks for reliable healthcare monitoring. IEEE Journal of Biomedical and Health Informatics, 18(5), 1541-1551. doi:10.1109/JBHI.2014.2312214 PMID:25192567 
Skubic, M., Guevara, R. D., \& Rantz, M. (2015). Automated health alerts using in-home sensor data for embedded health assessment. IEEE Journal of Translational Engineering in Health and Medicine, 3, 1-11. doi:10.1109/ JTEHM.2015.2421499 PMID:27170900

Tizhoosh, H. R. (2005, November). Opposition-based learning: a new scheme for machine intelligence. In Proceedings of the Computational intelligence for modelling, control and automation, 2005 and international conference on intelligent agents, web technologies and internet commerce, international conference on (Vol. 1, pp. 695-701). IEEE. doi:10.1109/CIMCA.2005.1631345

Tóth-Laufer, E., \& Várkonyi-Kóczy, A. R. (2014). A soft computing-based hierarchical sport activity risk level calculation model for supporting home exercises. IEEE Transactions on Instrumentation and Measurement, 63(6), 1400-1411. doi:10.1109/TIM.2014.2299523

Wang, B., Jin, X., \& Cheng, B. (2012). Lion pride optimizer: An optimization algorithm inspired by lion pride behavior. Science China. Information Sciences, 55(10), 2369-2389. doi:10.1007/s11432-012-4548-0

Yazdani, M., \& Jolai, F. (2016). Lion optimization algorithm (LOA): a nature-inspired metaheuristic algorithm. Journal of computational design and engineering, 3(1), 24-36.

Yuan, R., Li, Z., Guan, X., \& Xu, L. (2010). An SVM-based machine learning method for accurate internet traffic classification. Information Systems Frontiers, 12(2), 149-156. doi:10.1007/s10796-008-9131-2

Zidi, S., Moulahi, T., \& Alaya, B. (2018). Fault Detection in Wireless Sensor Networks through SVM Classifier. IEEE Sensors Journal, 18(1), 340-347. doi:10.1109/JSEN.2017.2771226

Dr. Madhumita Kathuria is an Assistant Professor in Computer Science and Engineering Department at Manav Rachna International University, Faridabad, India. She is pursuing her PhD (Computer Science \& Engineering) from YMCA University of Science and Technology. She has published more than 15 papers in various international and national journals and conferences. Her area of interests includes wireless body area network, sensor network, network security, digital image processing, learning and computational techniques.

Dr. Sapna Gambhir is Assistant Professor in Computer Engineering Department in YMCA University of Science and Technology, Faridabad, India. She has done her PhD at Jamia Millia Islamia University in 2010. She has published more than 50 papers in various national and international journals and conferences. Her area of interests includes wireless sensor network, ad-hoc network and social network, and security of wireless networks. 